\title{
Optical Nanoprobing via Spin-Orbit Interaction of Light
}

\author{
K. Y. Bliokh ${ }^{1}$, O. Rodríguez-Herrera ${ }^{1}$, D. Lara ${ }^{2}$, E. A. Ostrovskaya ${ }^{3}$, C. Dainty ${ }^{1}$ \\ ${ }^{1}$ Applied Optics, School of Physics, National University of Ireland, Galway, Galway, Ireland \\ ${ }^{2}$ The Blackett Laboratory, Imperial College London, SW7 2BW, London, United Kingdom \\ ${ }^{3}$ ARC Centre of Excellence for Quantum-Atom Optics and Nonlinear Physics Centre, Research School of Physics \\ and Engineering, The Australian National University, Canberra ACT 0200, Australia
}

(Invited Paper)

\begin{abstract}
High-NA optical microscopy is accompanied by strong spin-orbit interaction of light, which translates fine information about the nano-size specimen to the non-uniform polarization distribution of the outgoing light. We observe angular momentum conversion and spin-Hall effect of light, which are highly sensitive to the position of the nanoparticle.
\end{abstract}

In the past few years spin-orbit interaction (SOI) of light was intensively studied in connection with the spin-Hall effect in inhomogeneous media [1,2] and conversion of the angular momentum (AM) of light upon focusing and scattering [3-5]. SOI phenomena exhibit intrinsic subwavelength-scale coupling between polarization and position of light. This makes them highly attractive for nano-optical applications, but difficult for detection in the paraxial far field. In isotropic media the subwavelength-scale SOI becomes conspicuous only in highly non-paraxial fields tightly focused by high numerical aperture (NA) lenses or scattered by small particles. In these cases, its observation requires additional interaction with testing particles [3] or near-field measurements [4,5].

Here we propose and demonstrate experimentally a far-field polarimetric imaging system that employs strong SOI of light and space-variant AM conversion via a nano-particle, Fig. 1 [6,7]. An $80 \mathrm{~nm}$ gold particle located near the focus between two high-NA lenses interacts with the non-paraxial focal field inside the system and dramatically modifies polarization distribution of the outgoing paraxial light.

The experimental and theoretical results are presented in Figs. 2 and 3 which clearly show characteristic nonuniform patterns in the output polarization [7]. First, when the scatterer is located precisely at the focal point and illuminated by circularly-polarized light, the Stokes parameters $S_{1}$ and $S_{2}$ possess four-fold symmetric patterns (Fig. 2) typical for the spin-to-orbit AM conversion of light upon scattering [8]. Strikingly, the sub-diffraction-limit displacement of the particle by the distance $\lambda / 3$ along, e.g., the $x$-axis induces dramatic changes in the output polarization patterns, breaking their symmetry along the orthogonal $y$-axis. Figure 3 shows drastic displacement-induced separation of the two spin states (positive and negative $S_{3}$ ) in the linearly $x$-polarized light, i.e., the giant spin-Hall effect of light. Instead of a tiny shift of the light position caused by switching between different spin states, which is typical for the usual spin-Hall effect $[1,2]$, we observe macroscopic redistribution of spins caused by the subwavelength displacement of the scatterer.
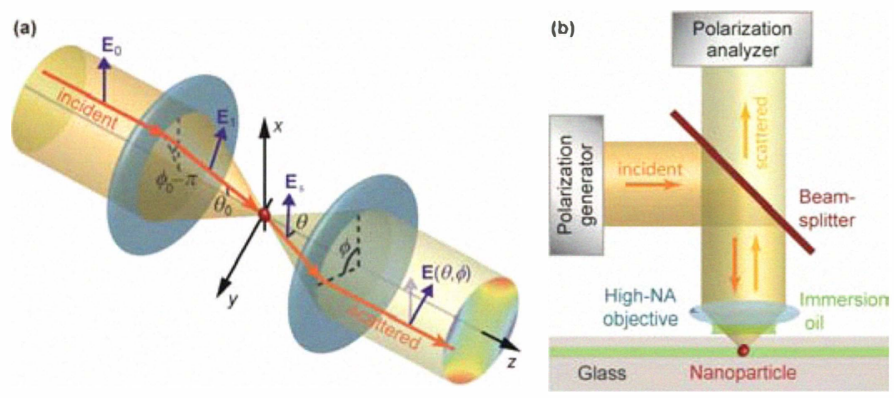

Fig. 1. Schematics of the microscopy of a nanoparticle, which involves the SOI of polarized light: (a) The "lensscatterer-lens" system underlying the theoretical model; (b) the experimental setup in the reflection configuration, where the focusing objective is also used as a collector lens and a beam splitter separates incident and scattered light.

We describe the system by means of a simple analytical model based on the geometrical transformations of light in the system. It relates the output space-variant polarization to the input state of light via the Jones matrix $\hat{J}\left(\theta, \phi, \rho_{\mathrm{s}}\right)$ which depends on the spherical angles of the exit pupil and dimensionless coordinates of the nano-particle, $\rho_{s}=k\left(x_{s}+i y_{s}\right)$. In the spin basis of circular polarizations it takes the form [7]

$$
\hat{J}=\frac{A}{\sqrt{\cos \theta}}\left(\begin{array}{cc}
a & -b e^{-2 i \phi} \\
-b e^{2 i \phi} & a
\end{array}\right)-i \frac{B \sin \theta}{2 \sqrt{\cos \theta}}\left(\begin{array}{cc}
\rho_{\mathrm{s}} e^{-i \phi} & \rho_{\mathrm{s}}^{*} e^{-i \phi} \\
\rho_{\mathrm{s}} e^{i \phi} & \rho_{\mathrm{s}}^{*} e^{i \phi}
\end{array}\right)
$$

where $A$ and $B$ are aperture-dependent constants, $a=\cos ^{2}(\theta / 2), b=\sin ^{2}(\theta / 2)$. Here the first term describes the spin-to-orbital AM conversion at $\rho_{s}=0$ (Fig. 2), whereas the second term accounts for the spin-Hall effect caused by the displacement of the particle (Fig. 3). 


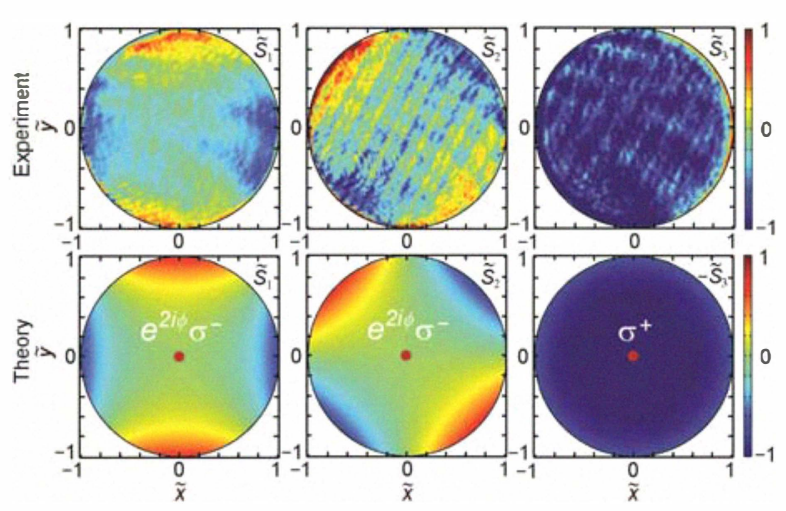

Fig. 2. Spin-to-orbital AM conversion of light when the nanoparticle is located precisely in the focal point. Experimentally measured and theoretically calculated from Eq. (1) distributions of the normalized Stokes parameters are shown for the case of circularly $R$-polarized incident light. We use normalized coordinates and the sign difference in $S_{3}$ between the theory and experiment arises from the helicity flip in the reflection configuration.

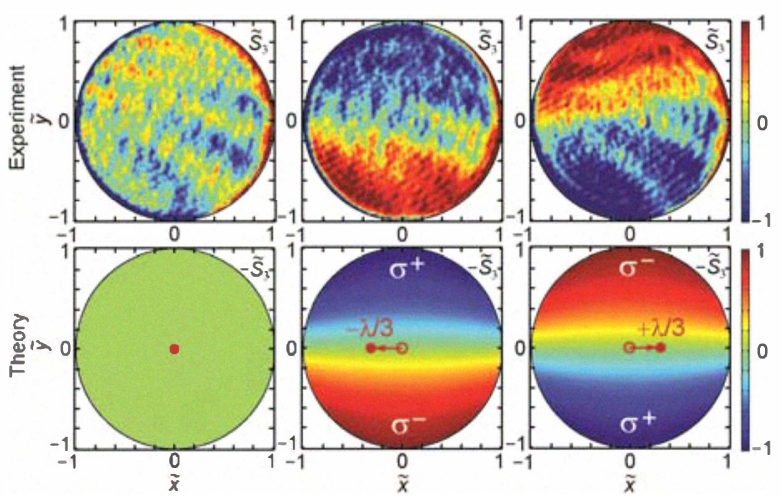

Fig. 3. Giant spin-Hall effect of light caused by subwavelength displacements of the nanoparticle. Experimentally measured and theoretically calculated, spatial distributions of the SAM density $S_{3}$ are shown for the case of linearly $x$-polarized incident light. Left-hand, middle, and right-hand panels correspond to the positions of the particle $x_{\mathrm{s}}=0,-\lambda / 3, \lambda / 3$, respectively.

To summarize, we have shown that far-field imaging of nanoobjects with polarized light is accompanied by strong SOI and AM conversion. This brings forth a new type of optical probing, whereby polarization degrees of freedom of light carry and reveal subwavelength information about the specimen.

\section{REFERENCES}

[1] P. Hosten and O. Kwiat, "Observation of the spin Hall effect of light via weak measurements," Science, vol. 319, pp. 787-790, 2008.
[2] K. Y. Bliokh, A. Niv, V. Kleiner, and E. Hasman, "Geometrodynamics of spinning light," Nature Photon., vol. 2, pp. 748-753, 2008.

[3] Y. Zhao, J. S. Edgar, G. D. M. Jeffries, D. McGloin, and D. T. Chiu, "Spin-to-orbital angular momentum conversion in a strongly focused optical beam," Phys. Rev. Lett., vol. 99, p. 073901, 2007.

[4] K.Y. Bliokh, Y. Gorodetski, V. Kleiner, and E. Hasman, "Coriolis effect in optics: Unified geometric phase and spin-Hall effect," Phys. Rev. Lett., vol. 101,p. 030404, 2008.

[5] D. Haefner, S. Sukhov, and A. Dogariu, "Spin Hall effect of light in spherical geometry," Phys. Rev. Lett., vol. 102, p. 123903, 2009.

[6] O. G. Rodríguez-Herrera, D. Lara, and C. Dainty, "Far-field polarization-based sensitivity to subresolution displacements of a sub-resolution scatterer in tightly focused fields," Opt. Express, vol. 18, pp. 5609-5628, 2010.

[7] O. G. Rodríguez-Herrera, D. Lara, K. Y. Bliokh, E. A. Ostrovskaya, and C. Dainty, "Optical nanoprobing via spin-orbit interaction of light," Phys. Rev. Lett., vol. 104, p. 253601, 2010.

[8] E. Brasselet, N. Murazawa, H. Misawa, and S. Juodkazis, "Optical vortices from liquid crystal droplets,” Phys. Rev. Lett., vol. 103, p. 103903, 2009. 